\title{
Sistema de Información Gerencial para la Administración de Recursos Educativos
}

\author{
Plinio Puello, Amaury Cabarcas y Raul J. Martelo \\ Universidad de Cartagena, Facultad de Ingeniería, Grupo de Investigación en Tecnologías de las \\ Comunicaciones e Informática, GIMATICA, Avenida del Consulado, Calle 30, No. 48 - 152, Cartagena- \\ Colombia. (e-mail: ppuellom@unicartagena.edu.co, acabarcasa@unicartagena.edu.co, \\ rmartelog1@unicartagena.edu.co )
}

Recibido Abr. 16, 2013; Aceptado May. 29, 2013; Versión final recibida Jul. 28, 2013

\begin{abstract}
Resumen
El objetivo del trabajo presentado fue proponer un enfoque para optimizar la administración de recursos educativos. La propuesta consiste en la inclusión de un sistema de información gerencial en la dinámica de utilización de los recursos para generar información que apoye a la toma de decisiones. Se determinó la utilidad de la propuesta mediante el diseño y construcción de una herramienta informática y su implementación en los laboratorios de computación de la Universidad de Cartagena en Colombia. El estudio mostró que el enfoque planteado permite ahorrar costos al realizar mantenimientos más efectivos de los recursos, evitar desabastecimiento y cuellos de botella en la dinámica de uso de los recursos y apoyar la toma de decisiones. Se concluye que la administración de recursos educativos se ve optimizada al implantar un sistema de información gerencial como el propuesto en este trabajo.
\end{abstract}

Palabras clave: sistemas de información gerencial, administración, recursos educativos, optimización, toma de decisiones

\section{Management Information System for Managing Educational Resources}

\begin{abstract}
The goal of proposed work was to propose an approach to optimize educational resources management. The proposal consists of the inclusion of a management information system within the dynamical use of these resources to generate information that support decision making. The utility of the proposal was determined through the design and implementation of a computer tool for the laboratories of the University of Cartagena in Colombia. The study showed that the proposed approach allowed saving costs with more effective resources maintenance, avoiding shortages and bottlenecks on the resources dynamic of use and supporting the decision making. It is concluded that educational resources management can be optimize by implementing a management information system as the one proposed in this work.
\end{abstract}

Keywords: management information system, management, educational resources, optimization, decision making 


\section{INTRODUCCIÓN}

Con el crecimiento de la tecnología en los últimos años los científicos han buscado la forma de aplicarla a cada área del conocimiento, así como también a cada proceso desarrollado en la sociedad. Los Sistemas de información gerencial no están exentos de esta tendencia, por el contrario, se han realizado múltiples trabajos de investigación para su aplicación en campos tan diversos como la optimización de portafolios de inversión (Martínez y Perozo, 2010), el mejoramiento de procesos en pequeñas y medianas empresas (Fernández y Plata , 2006), agencias de telecomunicaciones (Khanna y Sharma, 2012), manejo de equipamiento médico (Núñez y Castro, 2011) hasta agricultura de precisión (Nikkila et al., 2010). En el área de la educación también han sido diseñados diversos enfoques para sacar provecho a las ventajas de los SIG (Martínez, 2006; Saadé, 2003; Palomo et al., 2007; Zhang y Duan, 2010; Hosseini et al., 2012; Cassidy, 2005); sin embargo a pesar de los trabajos realizados, ninguno se ha enfocado directamente en el problema la optimización de la administración de recursos educativos, ya que a esta no se le presta la suficiente atención (Hua y Herstein, 2003); no obstante constituye uno de los mayores retos de la instituciones de educación públicas (Ogawa, 2004). Adicionalmente cuando la administración de recursos educativos no se lleva a cabo de forma adecuada puede causar la disminución de la economía y crecimiento de la pobreza (Jungand y Thorbecke, 2001).

Por lo anterior proponer un enfoque para solucionar el problema planteado mediante la implantación de un sistema de información gerencial en la dinámica de uso de los recursos educativos constituye el objetivo principal de este artículo. Para alcanzar el mismo se desarrolló un modelo conceptual de administración de recursos educativos, el cual consiste en la inclusión de un SIG en dicho proceso con el fin de apoyar la toma de decisiones tanto en el sentido de la asignación óptima de recursos como al control operacional del proceso. Con el fin de validar la efectividad del enfoque propuesto se realizó un experimento aplicado a la Universidad de Cartagena; este consistió en diseñar e implementar un SIG que brinde soporte la dinámica del acceso a recursos tecnológicos en los laboratorios de computación de dicha Universidad. Como principal resultado se encontró que el enfoque propuesto presenta ventajas que van desde el ahorro en costos de mantenimientos hasta el apoyo en la gestión de inventarios, entre otras soluciones desarrolladas; permitiendo soportar la efectividad y utilidad del SIG.

\section{FUNDAMENTOS TEORICOS}

La teoría general de sistemas o teoría de sistemas (TGS) es un esfuerzo de estudio interdisciplinario que trata de encontrar las propiedades comunes a entidades, los sistemas, que se presentan en todos los niveles de la realidad, pero que son objeto tradicionalmente de disciplinas académicas diferentes. Su puesta en marcha se atribuye al biólogo austriaco Ludwing Von Bertalanffy, quien acuñó la denominación a mediados del siglo XX (Capra, 1998; Bertalanffy, 1968). Fue la TGS quien planteó las bases para que las organizaciones fuesen tratadas como sistemas. Una organización es un sistema socio-técnico incluido en otro más amplio que es la sociedad con la que interactúa influyéndose mutuamente. De esta manera la empresa se presenta como una estructura autónoma con capacidad de reproducirse y puede ser visualizada, a través de una teoría de sistemas capaz de propiciar una visión de un sistema de toma de decisiones, tanto desde el punto de vista individual como colectivo; toda vez que la toma de decisión será mucho más fácil, si se cuenta con una descripción concreta y objetiva del sistema dentro del cual debe ser tomada. (Chiavenato, 1999).

La utilización de SIG para apoyar la toma de decisiones ha sido aplicada a gran variedad de campos del conocimiento desde diferentes enfoques. En (Fernández y Plata, 2006) se analizan los SIG que se requieren en las pequeñas y medianas empresas (Pymes) en el marco de la creación de entornos de éxito en tiempos de crisis. Por otro lado (Nikkila et al., 2010) identifica los requerimientos que deben tener los sistemas de información gerencial agrícola para ser aplicados al emergente campo de la agricultura de precisión. De otro lado Núñez y Castro (2011) proponen el desarrollo de SIG basado en dispositivos móviles para gestión de equipamiento médico. Además en (Khanna y Sharma, 2012) se presenta un enfoque para la implementación de SIG en empresas de telecomunicaciones para la agilización de procesos. Finalmente Martínez y Perozo (2010) propone la implementación de SIG en la optimización de portafolios de inversión administrados por la sociedad de carretaje de títulos valores en la bolsa de valores de Caracas.

En el campo de la educación también se han desarrollado múltiples trabajos que buscan sacar provecho de las ventajas de los SIG. En (Martínez, 2006) se da a conocer el diseño de un SIG integrado para la transformación de la gestión universitaria, con miras a mejorar su eficiencia, eficacia y pertinencia social. Así mismo $\mathrm{Ng}$ et al. (2011) presenta un enfoque para la toma de decisiones en la asignación de recursos y control operacional mediante un SIG de procesos educativos basado en plataforma web. Por otro lado en (Hosseini et al., 2012) se investigan las barreras para la utilización de SIG en las instituciones de educación superior mediante un enfoque de toma de decisiones multi criterio, presentando el caso de estudio de la 
Universidad Ferdowsi en Masshad. Por su parte Ji (2012) da a conocer un SIG para la administración de la educación llamado EMIS por sus siglas en inglés, el cual mediante el análisis de los datos suministrados ayuda a los sistemas escolares en las zonas rurales y remotas del suroeste de china con la planificación y gestión educativa. En el mismo sentido en (Echeverría et al., 2012) se presenta un SIG para la gestión de la educación diseñado para el departamento de planeación institucional y desarrollo de la Universidad de Colima. Todos estos referentes permiten seleccionar el enfoque de un SIG para abordar el problema planteado.

\section{DESARROLLO DEL ESQUEMA DEL SIG}

En esta sección se presenta el enfoque propuesto para abordar el problema de la administración de recursos educativos mediante un sistema de información gerencial. La figura de abajo presenta el modelo conceptual planteado.

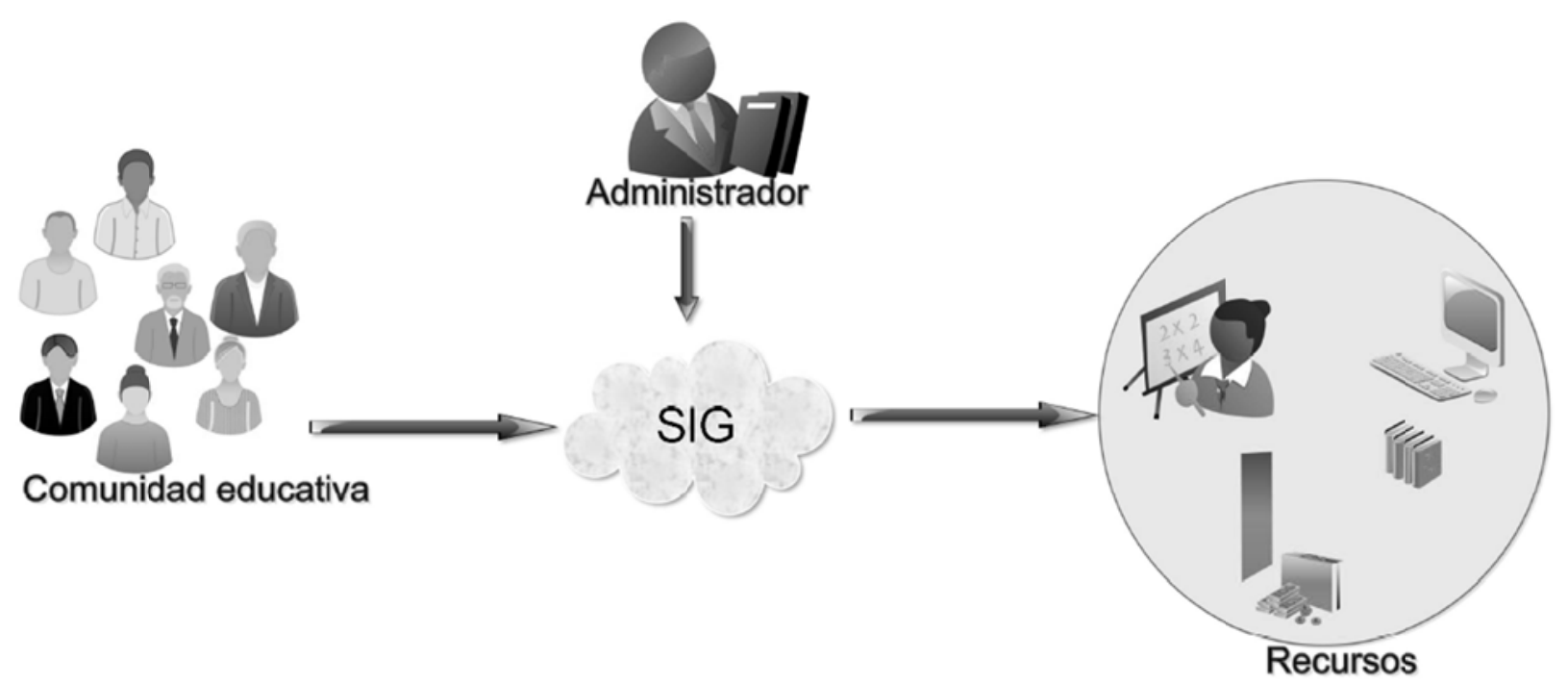

Fig. 1: Enfoque conceptual planteado.

En la figura de arriba se puede apreciar que el acceso a los recursos por parte de la comunidad educativa, se lleva a cabo a través de un SIG, el cual es manejado por un administrador. Con este enfoque se busca evitar el acceso directo y desordenado a los recursos, puesto que de esta manera no se saca provecho de la información que se puede obtener como resultado de la dinámica del mismo. Dado que toda la información del proceso de acceso a los recursos educativos se lleva a cabo mediante el SIG, este puede brindar al administrador información valiosa para facilitar la toma de decisiones, tanto en el aspecto de asignación de recursos como en el control operacional.

Con el fin de determinar la validez del enfoque propuesto se realizó el diseño e implementación de un SIG. Los indicadores de gestión definidos para el mismo fueron los siguientes:

Recursos con mayor utilización por categorías (dependiendo de rango de fechas)

Recursos con mayor utilización (dependiendo de rango de fechas)

Recursos sub utilizados (dependiendo de rango de fechas)

Personas con mayor frecuencia de uso (dependiendo de rango de fechas)

Fechas de mayor uso en el periodo académico de los recursos.

Record de uso por equipo

Adicionalmente se especificaron los siguientes requerimientos funcionales para la implementación del SIG: Generar reportes estadísticos de los indicadores definidos, para dar soporte a la toma de decisiones.

Gestionar la dinámica del uso de los recursos educativos.

Así con el fin de atender los requerimientos funcionales expuestos arriba, se diseñó la arquitectura que se aprecia en la figura 2. 


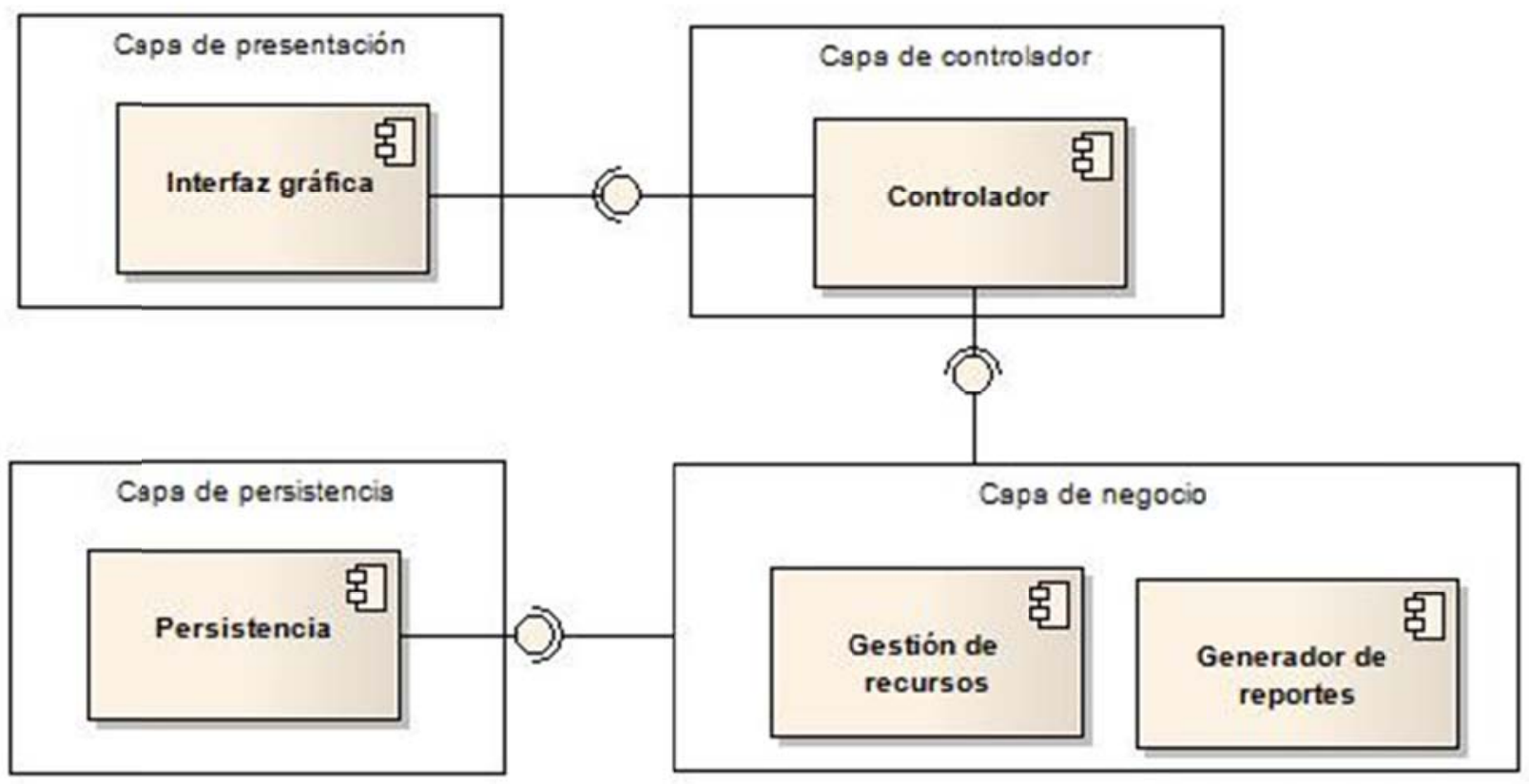

Fig. 2: Arquitectura del sistema de información gerencial.

Como se aprecia en la figura de arriba el SIG diseñado tiene una arquitectura de 4 capas. En la capa de presentación se encuentra el componente relacionado con el manejo de la interfaz gráfica de usuario, los eventos y acciones que se llevan a cabo en la misma son soportadas por la capa de controlador, quien los procesa y envía a la capa de negocio; en esta se encuentran dos componentes: por un lado se tiene el módulo relacionado con la gestión de recursos educativos, el cual almacena su información en un sistema de gestión de bases de datos (SGBD) mediante el módulo de la capa de persistencia, la que es a su vez la cuarta capa de la arquitectura. Finalmente el componente de negocio llamado Generador de reportes recupera esta información haciendo uso de la capa anterior y la procesa para proporcionar reportes con información gerencial, para dar apoyo a la toma de decisiones. Con este diseño se pretende obtener el mayor provecho de la arquitectura modelo vista controlador (MVC) en conjunto con una capa adicional de persistencia.

Por otro lado se considera pertinente precisar la necesidades y fuentes de información del SIG, por lo cual se resalta que las mismas fueron obtenidas utilizando un instrumento para recolección de información primario tipo encuesta, la cual fue aplicada a funcionarios encargados del manejo de recursos en la facultad quienes representan la totalidad del recurso humano que atiende a la Facultad de Ingeniería y de Ciencias, estos a su vez constituyen las fuentes de información primarias además de los formatos que utilizan dichos empleados en su labor diaria. La encuesta en mención fue concebida con el objetivo de medir las variables de atención a usuarios, capacidad de oferta y demanda de recursos y existencia de herramientas para la generación de reportes para soportar procesos académicos y administrativos. La información identificada a través de los instrumentos de recolección permitió alimentar los elementos organizativo, administrativo y tecnológico que constituyen el sistema de información gerencial para la gestión de recursos educativos. La lógica plasmada en el modelo de negocio se produjo al analizar las diversas situaciones del mundo real y proponer un cambio tecnológico al proceso actual realizado de manera manual utilizando medios físicos.

Una de las necesidades encontradas se tiene que el actual proceso de reserva y préstamo de recursos educativos es realizado en medio físico por medio de formatos destinados para la asignación de cada recurso. La mayor necesidad en este proceso se identificó al momento de realizar el préstamo de recursos y generar reportes para medición de atención a usuarios, capacidad de oferta y demanda de recursos ya que se debe revisar manualmente los formatos diligenciados por cada equipo o recurso (existiendo una hoja por día para cada uno de ellos) para realizar el proceso de préstamo. Igualmente al requerir la generación de reportes de atención a usuarios se debe ir totalizando de forma manual y tediosa la información solicitada en cada reporte, presentándose en algunas ocasiones errores humanos durante el conteo físico de los formatos; así mismo las demoras en la atención y falta de mecanismos para obtener información gerencial de la dinámica del proceso que facilite la toma de decisiones constituyen necesidades importantes para la prestación del servicio. 
De otro lado el despliegue del sistema se organiza de la siguiente manera:

Software de Cómputo: Se utilizaron aplicativos de desarrollo basado en licencia de software libre y se instaló el servidor de publicación del servicio en uno de los equipos de cómputo asignados en una de las ubicaciones físicas donde funciona las oficinas para el préstamo de los recursos educativos.

Tecnología de administración de datos: Se utilizó un gestor de bases de datos basado en software libre como lo es PostgreSQL.

Tecnología de Conectividad de redes y telecomunicaciones: Se utilizó la infraestructura para conectividad de los espacios y edificios de la Universidad de Cartagena Sede Piedra de Bolívar.

En este esquema el mecanismo de control y seguridad utilizado consiste en el manejo de perfiles y roles de usuarios con el fin de poder auditar en cualquier momento las acciones realizadas, y de esta manera establecer responsabilidades en caso de anormalidades en la dinámica del proceso.

Por otro lado se considera que la implementación del sistema en los laboratorios de cómputo de la Universidad de Cartagena tiene un impacto que cobija toda la comunidad estudiantil de esta institución y muchos de sus docentes, lo que constituye una población de aproximadamente de 11000 personas.

\section{RESULTADOS Y DISCUSIÓN}

Con el fin de validar la efectividad del enfoque planteado se simuló un escenario como el de la figura 1 en los laboratorios de computación del programa de Ingeniería de Sistemas de la Facultad de Ingeniería de la Universidad de Cartagena, en el que se utilizó el SIG diseñado para el acceso a los recursos educativos. A continuación se presentan los resultados del experimento realizado teniendo en cuenta los indicadores definidos en la sección anterior:

En primer lugar el indicador "Recursos con mayor utilización por categorías" permitió conocer el grupo de recursos con mayor rotación, lo cual dio a conocer patrones de uso por parte de la comunidad educativa. La figura 3 corresponde al reporte generado por la herramienta diseñada para el indicador en cuestión:

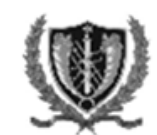

Universidad de Cartagena

Facultad de Ingenieria

Programa de Ingenieria de Sistemas

Rango de fechas: de 02/04/2013 a 05/04/2013

Recursos con mayor utilización por categorias

\begin{tabular}{lll}
\hline \multicolumn{2}{|c}{ Recursos con mayor utilización por categorias } \\
\hline Nombre & Descripción & Número de préstamos \\
\hline Computador & Equipo para prácticas de laboratorio & 8 \\
Servidor & Servidor de datos & 5 \\
Enrutador & Equipo Cisco serie 3900 & 3 \\
Teléfono NFC & Nokia C7 para pruebas NFC & 3 \\
Impresora & Equipo para impresión de trabajos. & 2 \\
Multimetro & Implemento para prácticas de electrónica. \\
Teléfono inteligente & Samsung Omnia & 2 \\
Portatil & Equipo para desarrollar investigación & 2 \\
\hline TOTAL PRESTAMOS: & & 26
\end{tabular}

Fig. 3: Reporte del indicador "Recursos con mayor utilización por categoría". 
Como puede apreciarse en la figura 3, los recursos más utilizados corresponden a los involucrados en el proceso de aprendizaje, mientras que los menos usados son aquellos necesarios en el proceso de investigación.

Por otro lado, con relación al indicador "Personas con mayor frecuencia de uso", se obtuvo resultados que permitieron determinar que la utilización de los recursos se lleva a cabo por un reducido número de personas, lo que indica que existen miembros de la comunidad educativa (no se presentarán datos precisos por cuestión de privacidad) que presentan alta demanda de recursos, mientras que otros no hacen uso de los mismos. Esta información puede utilizarse para determinar responsabilidades en caso de presentarse daños en los recursos, o para detectar individuos con necesidades especiales de aprendizaje. De otro lado el indicador "Recursos con mayor utilización" arrojó entre otras cosas que el recurso más utilizados es el computador de número de serie 567, seguido por el seriado 114 y el 12 respectivamente; esta información es similar a la presentada por el "Recursos con mayor utilización por categoría" con un matiz interesante, ya que al conocer los recursos individuales que reciben mayor carga de uso se pueden programar mantenimientos selectivos a dichos recursos (con el ahorro económico que ello sugiere), esta información complementa de forma significativa la arrojada por el indicador que se analizó en primera instancia en esta sección.

Otro indicador analizado fue "Fechas de mayor uso en el periodo académico de los recursos" mediante el cual se pudo establecer que fechas como el 04 de abril presentan mayor número de préstamos, lo cual se debe posiblemente al retorno de los miembros de la comunidad educativa a actividades luego del receso de las vacaciones cortas. Lo que permite establecer estrategias en términos potencialización de inventarios para las temporadas del retorno académico y adquisición de personal de apoyo. Finalmente mediante el resto de indicadores se encontró que recursos como portátiles y teléfonos inteligentes son utilizados escasamente lo que sugiere directrices en cuanto a la gestión de inventarios. De los resultados anteriores se puede deducir que el enfoque planteado es útil para optimización de la administración de recursos educativos.

\section{CONCLUSIONES}

De los resultados y el análisis presentado se pueden obtener las siguientes conclusiones sobre el enfoque propuesto y la herramienta que lo soporta: 1) La administración de recursos educativos se ve optimizada al implantar un sistema de información gerencial que soporte la dinámica del proceso. 2) Permite determinar patrones de uso de los recursos educativos. 3) Ayuda a establecer responsabilidades en caso de averías de equipos con el consecuente ahorro que ello supone. 4) Da a conocer las fechas críticas para evitar desabastecimiento y cuellos de botella en la prestación del servicio. 5) Apoya la toma de decisiones en el manejo del inventario de recursos. 6) Permite ahorrar costos al realizar mantenimientos más efectivos de los recursos. 7) Cualquier proceso de innovación tecnológica tiene muchos beneficios proyectados a corto, mediano y largo plazo, que al materializarse a través del uso este tipo de sistemas en las organizaciones permiten que los procesos cotidianos generen sinergia en la compañía, es decir, se aumente los niveles de integración y se tomen decisiones acertadas y ajustadas a la realidad.

\section{REFERENCIA}

Bertalanffy, L. V. Teoría General de los Sistemas. 7a reimpresión 1989, 311 págs., Fondo de Cultura Económica, México, (1968).

Capra, F. La trama de la vida. Barcelona: Editorial Anagrama, (1998).

Cassidy, T. Education Management Information System (EMIS) Development in Latin America and the Caribbean: Lessons and Challenges.Tersedia: Acceso: http://www. iadb. org/IDBDocs. cfm (2005).

Chiavenato, I. Introducción a la teoría general de la administración. Santafé de Bogotá: McGraw-Hill, (1999).

Echeverría, M. A. M., Santana-Mancilla, P. C., y Cazares, V. M. D. L. R. An Educational Management Information System to Support Institutional Planning at the University of Colima, Procedia-Social and Behavioral Sciences: 55, 1168-1174 (2012).

Fernández, L. S., y de Plata, D. P. Los sistemas de información gerencial en las PYMEs en el marco de la creación de un entorno de éxito en tiempos de crisis, Multiciencias:1, (2006).

Hosseini, M. H., Karimzadegan, D., y Sazvar, A. Identification of Management Information System (MIS) strategies barriers in higher education institutions through multi-criteria decision making (MCDM) approach: Case study of Ferdowsi University of Mashhad, Educational Research and Reviews: 7(5), 111-120 (2012). 
Hua, H., y Herstein, J. Education management information system (EMIS): Integrated data and information systems and their implications in educational management, In annual conference of comparative and International Education Society: (2003).

Ji, J., y Lai, F. Q. EMIS Data Analyzing and Monitoring for Educational Planning. In World Conference on Educational Multimedia, Hypermedia and Telecommunications: 2012(1), 179-182 (2012).

Jung, H. S., y Thorbecke, E. The impact of public education expenditure on human capital, growth, and poverty in Tanzania and Zambia: a general equilibrium approach, Journal of Policy Modeling: 25(8), 701-725 (2003).

Khanna, S., y Sharma, A. Role of Management Information System in Telecom Agencies, IJCAIT: 1(1), 1-3 (2012).

Martínez, H. R., y Perozo, B. J. Sistema de información gerencial para la optimización de portafolios de inversión, Revista Venezolana de Gerencia: 15(50), 253-272 (2010).

Martínez, R. Estudio y diseño de un sistema de información gerencial universitario integrado (SIGUI) (2006).

Ng, S. C., Wong, C. K., Lee, T. S., y Lee, F. Y. Design of an Agent-based Academic Information System for Effective Education Management. Information Technology Journal: 10(9), 1784-1788 (2011).

Nikkilä, R., Seilonen, I., y Koskinen, K. Software architecture for farm management information systems in precision agriculture, Computers and electronics in agriculture: 70(2), 328-336 (2010).

Núñez, C., y Castro, D. Management information system of medical equipment using mobile devices, In Journal of Physics: Conference Series: 313(1), (2011).

Ogawa, K. Public expenditure on education and resource management: Case of Zambia. Journal of International Cooperation in Education: 7(1), 133-143 (2004).

Palomo, I. F., Veloso, C. G., y Schmal, R. F. Sistema de Gestión de la Investigación en la Universidad de Talca, Chile, Información tecnológica: 18(1), 97-106 (2007).

Saadé, R. G. Web-based educational information system for enhanced learning, EISEL: Student assessment. Journal of Information Technology Education: 2, 267-277 (2003).

Zhang, M., y Duan, R. Design of Web based Management Information System for Academic Degree \& Graduate Education. In IFIP International Federation for Information Processing: 252(1), (2010). 
